\title{
Activités
}

\section{Analyse collective du travail des inspecteurs des impôts au Brésil dans l'État de Rio de Janeiro}

Collective analysis of the work of Brazilian tax auditors in the state of Rio de Janeiro

Roberto Bartholo, Rita de Cássia Monteiro Afonso, Arminda Eugenia Marques Campos, Gabriel Bursztyn et Maurício César Delamaro

\section{OpenEdition}

\section{Journals}

Édition électronique

URL : http://journals.openedition.org/activites/1035

DOI : 10.4000/activites. 1035

ISSN : 1765-2723

Éditeur

ARPACT - Association Recherches et Pratiques sur les ACTivités

Référence électronique

Roberto Bartholo, Rita de Cássia Monteiro Afonso, Arminda Eugenia Marques Campos, Gabriel Bursztyn et Maurício César Delamaro, « Analyse collective du travail des inspecteurs des impôts au Brésil dans l'État de Rio de Janeiro », Activités [En ligne], 12-1 | Avril 2015, mis en ligne le 15 avril 2015, consulté le 19 avril 2019. URL : http://journals.openedition.org/activites/1035; DOI : 10.4000/ activites. 1035

\section{(c) (i) (9)}

Activités est mis à disposition selon les termes de la licence Creative Commons Attribution - Pas d'Utilisation Commerciale - Pas de Modification 4.0 International. 


\title{
Analyse collective du travail des inspecteurs des impôts au Brésil dans l'État de Rio de Janeiro \\ Roberto Bartholo
}

Programa de Engenharia de Produção - COPPE/UFRJ (Universidade Federal do Rio de Janeiro). bartholo.roberto@gmail.com

\section{Rita de Cássia Monteiro Afonso}

Faculdade de Administração e Ciências Contábeis - FACC /UFRJ (Universidade Federal do Rio de Janeiro) ritaafonso@globo.com

\section{Arminda Eugenia Marques Campos}

Faculdade de Engenharia de Guaratinguetá - FEG / UNESP (Universidade Estadual Paulista). arminda.campos@feg.unesp.br

\section{Gabriel Bursztyn}

CITU (Cybermédia, Interaction, Transdisciplinarité, Ubiquité) - Université Paris 8 - Vincennes - Saint-Denis. gbursztyn@gmail.com

\section{Maurício César Delamaro}

\begin{abstract}
Faculdade de Engenharia de Guaratinguetá - FEG / UNESP (Universidade Estadual Paulista).
\end{abstract} delamaro@feg.unesp.br

\begin{abstract}
Collective analysis of the work of Brazilian tax auditors in the state of Rio de Janeiro. The article presents the results of a study of tax inspectors from Brazil's Receita Federal (tax administration). It draws on contributions made by Dejours (1980) and Gaulejac (2005b) on the alignment of public administration and management. It highlights the inspectors' dissatisfaction and suffering in relation to the changes implemented by the department's senior management as part of the "Modernizing Brazil" project.

KEYWORDS

management and public administration in Brazil, collective analysis of work, subjectivity in the workplace, tax inspectors work, public officials dissatisfaction with work
\end{abstract}

\section{1.- Introduction}

Cette étude présente les résultats d'une analyse collective du travail des inspecteurs des impôts au Brésil, en activité dans l'État de Rio de Janeiro. La recherche s'est efforcée de procéder méthodologiquement en accord avec la proposition de Dejours d'une psychodynamique du travail, en ayant comme centre prioritaire d'attention «la compréhension des rapports dynamiques entre le bien-être, un équilibre instable et la souffrance » (Maranda, 1995, p. 233). Dans notre étude, nous développons une analyse dynamique des processus psychiques mobilisés dans la confrontation du sujet collectif avec les situations de travail. Nous considérons que le collectif de travail ne se réduit pas à une 
somme de personnes regroupées par une volonté extérieure, mais qu'il se constitue dans le contexte d'une "communauté d'appartenance, source d'identification et de reconnaissance sociale, qui travaille dans le respect des règles et de la coopération à une œuvre commune » (Maranda 1995, p. 227).

Le syndicat des inspecteurs des Impôts de la Receita Federal (l'administration fiscale) UNAFISCO Rio, en s'adressant au Laboratório de Tecnologia e Desenvolvimento Social (Laboratoire de Technologie et de Développement Social) du Programme de Master et de Doctorat en Ingénierie de Production de l'Université Fédérale de Rio de Janeiro (UFRJ) cherchait à définir une position par rapport à un phénomène observé : dans les années récentes, le nombre de congés pour raison de santé de ses membres a sensiblement augmenté. Selon l'UNAFISCO Rio, le nombre de cas aurait plus que doublé en cinq ans.

L'accès à la carrière d'inspecteur des Impôts est très difficile. On y entre par concours public avec un salaire relativement élevé par rapport au niveau de vie brésilien ${ }^{1}$. La Receita Federal est un organisme gouvernemental qui relève du ministère des Finances, responsable de la collecte des principaux impôts fédéraux au pays. Les inspecteurs des impôts sont responsables des activités suivantes: l'exécution des obligations fiscales par les contribuables; la perception des impôts ; le contentieux fiscal ; le contrôle des douanes du commerce extérieur ; la gestion technique et administrative des bureaux de l'organisation; et les services aux contribuables individuels et aux représentants d'entreprises. Les activités menées par les inspecteurs sont donc très diverses. À l'époque de la réalisation de la recherche, les membres de l'UNAFISCO Rioreprésentaient presque $90 \%$ du total de la catégorie professionnelle.

Dans la période qui précède la recherche, les principaux changements subis par les personnels dans leuractivité correspondaient à une pression accrue pour augmenter les recettes fédérales et la mise en place, par la direction générale de la Receita Federal, de systèmes d'évaluation de la performance basés sur des objectifs quantitatifs rigides, pour toute l'organisation, pour chaque secteur et pour chaque individu.

Le Syndicat National des Inspecteurs des Impôts de la Receita Federal avait identifié dans son secteur un désarroi de la profession quant à son rôle public et son identité et quant au niveau de rendement à laquelle ses membres étaient soumis. Pour le syndicat, ces questions seraient associées à des changements dans l'administration de l'institution, soutenus par le discours qui affirme que cette dernière serait au service d'un "Brésil moderne », entraînant, dans les faits, une série de conséquences ressenties par les personnels comme néfastes au développement de leurs fonctions.

Le principal changement fut l'introduction d'un nouveau «modèle de gestion par performance », avec l'établissement d'ambitieux objectifs quantitatifs et le contrôle strict de son accomplissement. Ces objectifs, irréalistes selon le syndicat, furent définis par la direction, sans dialogue préalable avec le syndicat. Ils établissent, par exemple, un nombre minimum d'infractions qui doivent être détectées, mais aussi que seuls les niveaux hiérarchiques les plus élevés peuvent désigner les dossiers devant faire l'objet d'un contrôle fiscal. Un contrôle ciblé est effectivement conçu par la direction par analyse statistique des cas justifiant de concentrer les forces de contrôle en fonction du rendement espéré. Pour le UNAFISCO Rio, il s'agit d'une «incitation à l'erreur», à partir du moment où, l'amélioration des statistiques de poursuite devenant la finalité ultime du contrôle fiscal, la poursuite d'infractions risque d'aller jusqu'à en inventer pour atteindre les objectifs préétablis.

Selon le syndicat, le nouveau modèle de gestion est apparu équivalent, pour les contrôleurs, à une perte d'autonomie dans leur activité. Le contrôle fiscal devenait une opération

1 Tandis que le salaire minimum brésilien, alors de 350 réals (soit 167,50 dollars américains), le salaire initial d'un inspecteur des Impôts s'élevait à 10.000 réals ou 4.784,69 dollars. 
restreinte à une pré-programmation établie. Ne pas suivre le programme impliquait pour les contrôleurs qu'ils soient la cible d'une enquête de la Corregedoria (organisme responsable de l'inspection de l'administration), ce qui augmentait les tensions associées à l'exercice de la profession.

C'est ainsi que le syndicat a sollicité une étude qui lui permettrait de soutenir des propositions d'amélioration pour la profession. La recherche a effectué un diagnostic des conditions de travail dans ce domaine, en distinguant des aspects relatifs à l'amélioration de la qualité de vie au travail des fonctionnaires, leur rapport avec leur propre image, et leur efficacité.

Deux enquêtes ont été menées au long du premier semestre de 2007: d'une part, un questionnaire sur le climat de l'organisation et d'autre part, une enquête qualitative sur le travail à partir du vécu des inspecteurs. Le sondage concernant le climat organisationnel a permis de rassembler des informations qui visent à mettre en contexte la situation de travail des professionnels étudiés. Nous présentons ici les principaux résultats de l'enquête qualitative réalisée à partir de la méthodologie d'Analyse Collective du Travail - ACT .

\section{2.- Cadre théorique}

Comme l'indique Perret (2009), dans l'analyse critique en «sciences de la gestion», la «fabrique du consentement ${ }^{2}$ et les difficultés rencontrées par les membres d'une organisation pour exprimer leurs désaccords sont un sujet central. Perret s'appuie sur l'œuvre classique de Marcuse (1964) ${ }^{3}$ pour critiquer la prétention de l'idéologie managériale à faire du consensus le moteur du changement et pour montrer comment les techniques de gestion servent à mener les individus à des «situations d'injonctions paradoxales » (Perret 2009). Dans ce contexte, les œuvres de Christophe Dejours (1998, 2011), en psychodynamique du travail, et Vincent de Gaulejac (2005a, 2005 b), en sociologie clinique, se distinguent parmi les références théoriques pouvant servir à une critique des «sciences de la gestion » contemporaines.

D'un point de vue de fabrique du consentement, l'autonomie du sujet est une question risquée et problématique. Comme le dit Guyon (2010), dans sa réponse à la question «Qui est le je ? »", Gaulejac considère que «le sujet advient à partir d'un déjà-là » via l'ordre symbolique dans lequel sont prises les relations sociales. Le psychisme et le social s'étayent alors dans « une relation systémique et récursive ». La perspective de Gaulejac affirme ainsi l'existence de deux pôles distincts à considérer: l'un psychique et l'autre social; tous les deux irréductibles et relativement indépendants, tous les deux contingents et instables (2005 b, 2005c). Dans ce contexte, la subjectivation est une recherche continuelle de médiations face à des contradictions dont l'infinie combinatoire dans les registres psychiques, sociaux et familiaux explique la singularité (Guyon, 2010). Dans un monde en changement permanent, le sujet est ainsi dissous dans une économie de conflits intrapsychiques.

La fabrique du consentement dans les organisations contemporaines impose par concession hétéronome aux personnes une pseudo-autonomie, qui les pousse à se soumettre à des « injonctions paradoxales» (Gaulejac, 2005 b ; Perret, 2009). Les rhétoriques humanistes et émancipatoires ne sont qu'écrans de fumée au service de mécanismes de manipulation et de contrôle. Pour Dejours (1998), la généralisation des injustices dans le monde contemporain

2 Titre de l'oeuvre de N. Chomsky et E. Herman, Manufacturing Consent, 1988.

3 V. Perret (2009) écrit: “à la fin des années 60, Herbert Marcuse, se pose en témoin (amer) de ce basculement: 'le peuple, auparavant le ferment du changement social, s'est élevé, il est devenu le ferment de la cohésion sociale'. (H. Marcuse, 1964)".

4 Voir V. Gaulejac (2009) 
repose sur le consentement construit par la domination symbolique dans un système où les dominés vivent effrayés et acceptent pour eux et pour les autres une souffrance qui devient indépassable. La peur et la honte sont les ressorts psychologiques de ce consentement (à subir et à infliger).

Pour Gaulejac : «L'entreprise propose à l'homme managérial de satisfaire ses fantasmes de toute-puissance et ses désirs de réussite, contre une adhésion totale et une mobilisation psychique intense. L'idéalisation et l'identification le mettent dans une dépendance psychique importante. (...) ce n'est plus un engagement réciproque qui règle les rapports entre l'individu et l'organisation, mais une injonction paradoxale. Plus il réussit, plus sa dépendance augmente»(Gaulejac, 2005b, p.91). Il souligne que dans la fabrique du consentement, la violence s'exprime beaucoup plus sous le visage plus feutré de la violence psychique et « les conflits se posent de moins en moins au niveau de l'organisation en termes de luttes revendicatives ou de respect de l'autorité hiérarchique. Ils se posent au niveau psychologique en termes d'insécurité, de souffrance psychique, d'épuisement professionnel, de troubles psychosomatiques, de dépressions nerveuses. Autant de conflits face auxquels les syndicats ou les contre-pouvoirs sont démunis » (Gaulejac, 2005b, pp. 95-96).

Pour E. Renault (2008), le manque d'analyse des dimensions sociales et organisationnelles de la souffrance n'est pas restreint aux sciences de la gestion. Il touche tout le champ des sciences sociales. La description des lieux de souffrance dans la vie de tous les jours est une tâche primordiale pour la pensée critique contemporaine. Ces descriptions peuvent aider les gens à agir collectivement. La pensée critique est face à un défi stratégique : la fabrique du consentement semble laisser impuissantes les stratégies collectives classiques de défense, comme le cas exemplaire des grèves. En résumé, l'identification de la souffrance comme symptôme est une question cruciale pour l'analyse critique contemporaine. Pour cela, il est primordial de ne pas «psychologiser» les problèmes sociaux : «s'il y a une genèse sociale des conflits psychiques, on ne peut pas traiter uniquement ces conflits au niveau psychique et intrapsychique. Il faut aussi se préoccuper des liens avec leur genèse sociale. Est-ce que c'est du domaine de la thérapie ou pas ?... Les psychothérapeutes sont dans un cadre dans lequel ils ne le peuvent pas ; ce cadre ne peut traiter que les effets psychiques de ces situations sociales. Il faut donc que ce cadre thérapeutique soit articulé à d'autres cadres, d'autres formes de travail qui prennent mieux en compte la dimension sociale des problèmes » (Gaulejac, 2005c, p. 103).

La souffrance au travail pour Dejours serait également à rattacher à l'organisation du travail coexistant, donc avec sa "normalité », face à laquelle l'individu développe des mécanismes de régulation, c'est-à-dire de défense. Ces défenses ne seraient pas individuelles, mais plutôt collectives, démontrant le besoin que le groupe sanctionne et approuve les mécanismes construits pour réguler la souffrance au travail. Les individus du collectif partagent les stratégies défensives et excluent ceux qui ne s'adaptent pas à elles. Tandis que les mécanismes individuels de défense se maintiennent à partir d'intériorisations, les idéologies défensives collectives se construisent à partir de faits concrets du contexte qui feraient en sorte, s'ils étaient déplacés, qu'elles s'annulent puisqu'elles ne seraient pas nécessaires comme mécanismes collectifs de défense (Dejours, 1995, 1998, 2011).

Ainsi, dans ce cadre on observe une disqualification des savoirs et des métiers imposée sous la primauté de la gestion. Du point de vue des travailleurs, l'évaluation quantitative s'associe à l'injustice et l'isolement, de telle sorte que la possibilité de «vivre ensemble » au travail est déstructurée. La dégradation de la santé mentale des travailleurs est le terreau propice aux actes extrêmes (jusqu'au suicide) (Dejours \& Buègue, 2009).

Il y a une « convergence des psychologisants » entre l'analyse de la souffrance au travail par Dejours (1980) et le refus de Gaulejac (2005a) d'identifier la souffrance en tant que «l'expression d'un phénomène névrotique individuel». Dans ce même contexte, Lacroix nous a indiqué les conséquences les plus destructives de la pseudo psychologisation : «nous 
avons vu que le développement personnel partait en guerre contre le sociologisme en affirmant que l'individu n'est pas soumis aux déterminismes de son milieu. Nous voyons maintenant l'aboutissement de cette démarche. C'est non seulement l'influence de la société, mais sa réalité même qui est niée. Le corps politique, les structures socioéconomiques sont désintégrés par la psychologisation » (Lacroix, 2004, p. 97).

Gaulejac (2005 b) présente la gestion comme un système d'organisation du pouvoir qui se serait répandu depuis les années 1980 dans le monde des grandes entreprises publiques et privées, en inventant des mesures (souvent opposées) afin de soumettre l'individu à la productivité nécessaire à la nouvelle division internationale du travail et en traduisant des activités humaines en chiffres de performances. Selon l'auteur, cette idéologie transforme l'être humain en ressources au service de l'organisation, par le biais de la gestion qui rend opérationnel un projet de "quantophrênie $»^{5}$, de sorte que se perdrait le sens de la mesure. Des discours liés aux processus de management sous-tendent cette idéologie et en particulier le discours sur la qualité, en faisant une utopie propre à mobiliser l'enthousiasme et le consensus malgré leur anachronisme. Sous l'empire de la quantophrênie, le serviteur ne s'intéresserait au servi - le citoyen - que dans l'exacte mesure où celui-ci lui permettrait d'accroître ses résultats quantitatifs. La banalisation du mal sous le masque des chiffres permet une perversité capable, selon Dejours (1980), de produire des souffrances et des peurs ébranlant la santé mentale. D'après Gaulejac (2008, p. 439), « la destruction n'est pas tant le fruit d'un comportement pervers que d'un choix stratégique, effet d'un pouvoir abstrait, éloigné du terrain, occupé par des personnes qui ne se sont jamais confrontées directement à celles qui en subissent les conséquences ».

Nous présenterons ensuite les résultats d'une analyse collective du travail des inspecteurs des impôts au Brésil, en activité dans l'État de Rio de Janeiro. Notre recherche s'est efforcée de procéder méthodologiquement en accord avec la proposition de Dejours d'une psychodynamique du travail, ayant comme centre prioritaire d'attention «la compréhension des rapports dynamiques entre le bien-être, un équilibre instable et la souffrance » (Maranda, 1995, p. 233). Dans notre étude, nous développons une analyse dynamique des processus psychiques mobilisés dans la confrontation du sujet collectif à la réalité situationnelle du processus de travail. Nous considérons que le collectif de travail ne se réduit pas à une somme de personnes regroupées par des exigences extérieures, mais se constitue par une «communauté d'appartenance, source d'identification et de reconnaissance sociale, qui travaille dans le respect des règles et de la coopération à une œuvre commune » (Maranda, 1995, p. 227).

\section{3.- Méthodologie}

Cette recherche se caractérise par sa nature exploratoire. Puisque nous ne connaissions pas auparavant les conditions dans lesquelles se déroulait le travail des professionnels concernés, son objectif principal était de systématiser des connaissances de la situation, à partir desquelles il serait possible de réaliser des enquêtes plus détaillées et d'élaborer des propositions d'intervention. Elle présente une approche qualitative, car il était essentiel d'envisager la possibilité de prendre en considération les perspectives des individus et de comprendre le contexte de leurs actes. En termes de méthode, elle s'est définie comme une étude de cas rétrospective, ce qui s'est justifié par le besoin d'étudier le phénomène dans un contexte où nous nous sommes proposé d'analyser la totalité d'une situation pour interpréter le phénomène dans sa complexité.

La méthode employée pour l'étude de cas, dont cet article présente les résultats,

\footnotetext{
«Expression caricaturale de l'illusion qui consiste à croire que la réalité pourrait être comprise et maitrisée à condition qu'on puisse la mesurer, en la découpant en petites particules auxquelles on attribuerait un coefficient». (Gaulejac, 2005b, p. 95)
} 
s'appelle « Analyse collective du travail ». Elle a déjà été utilisée avec succès au Brésil. Des résultats de son application dans le pays ont été par ailleurs publiés (Ferreira, 1998 ; Ferreira, Bussacos, Schlithler, Maciel, \& Miyashita, 1992 ; Ferreira \& Donatelli, 2002 ; Ferreira \& Iguti, 1996 ; Hallak \& Silva, 2005 ; Ribeiro, 1992). L'analyse collective du travail est une occasion de se pencher sur ce qui n'est presque jamais formalisé : ce que chacun fait dans le travail. Parler, discuter et réfléchir à propos de son propre travail, c'est une façon de mieux le comprendre, un chemin vers une production de connaissances pour les travailleurs, les chercheurs et les autres sujets engagés dans la situation (Dejours, Abdoucheli, \& Jayet, 1994).

La recherche rejoint une perspective qui accorde la priorité à la distinction entre l'organisation du travail prescrit et le travail réel, c'est-à-dire le formel et le vivant. Cela implique de reconnaître que les stratégies «de défense» des travailleurs ne sont pas strictement défensives. Ce sont aussi des «pratiques de travail orientées vers la recherche de solutions face à une organisation incohérente et inefficace»(Brun, 1992), pratiques qui englobent en elles-mêmes « l'affrontement de défis, la création d'inédits, la mise en place de rapports de coordination, de coopération et de confiance qui s'observent dans les collectifs de travail » (Maranda 1995, p. 234). Comme l'affirment Cru et Dejours (1983), les règles de métier sont un art de vivre et montrent que le métier n'est pas seulement une question de technique. Pour la psychodynamique du travail, l'art de vivre des collectifs de travail met en œuvre des savoirs pratiques avec un objectif capable d'intégrer la qualité du travail et sa propre santé mentale (Dejours, 1995, 2011).

La méthode est basée sur le présupposé qu'il est possible de bien connaître le travail réalisé à partir de ce qu'en disent les gens qui l'exécutent. Elle consiste à réunir un groupe de travailleurs, volontaires, qui expliquent aux chercheurs leurs activités, c'est-à-dire ce qu'ils font dans le quotidien du travail. En voici les points principaux :

— le travail se déroule en groupe et non pas individuellement;

— tous les participants sont volontaires ;

— les réunions sont faites hors du lieu de travail ;

— au moins deux chercheurs mènent les réunions ;

— la question conductrice est «qu'est-ce que vous faîtes ?», à laquelle les interviewés doivent répondre de la façon la plus complète possible ;

— l'anonymat des travailleurs est garanti.

La recherche a débuté en février 2007 avec 52 participants, tous affiliés au syndicat UNAFISCO - Rio, regroupés selon la variété des fonctions exercées. Les participants avaient des temps de service variés dans la Receita Federal (de fonctionnaires récents ayant deux ans de services jusqu'à des retraités). La distinction des participants par sexe présenta une nette prédominance masculine (de l'ordre de $75 \%$ ). Le principal créneau d'âge représenté par l'échantillon était entre 40 et 45 ans (de l'ordre de $60 \%$ ). Ils ont été partagés en groupes d'inspecteurs qui, sous invitation du syndicat, étaient disposés à parler de leur activité. Six réunions ont été réalisées avec des individus regroupés selon le type de travail exercé : douane, contrôle contre la fraude, collecte des impôts, gestion, etc.

Les réunions se sont tenues au siège du syndicat en présence de seulement deux chercheurs et ont démarré avec la présentation des objectifs du projet et de la réunion. Après cette étape rapide, des questions-clés ont été posées «que vous faîtes-vous? », « décrivez votre journée de travail ». Les inspecteurs ont alors décrit ce dernier. Des questions des chercheurs et des autres participants ont aidé à la construction de connaissances sur les vécus. Le but était de parvenir à la description collective du travail dans chacun des groupes formés, d'après les similitudes de leurs activités. C'est la relation subjective entre enquêteurs et enquêtés qui a permis la construction de la connaissance du travail des inspecteurs de la Receita Federal.

Le local de réunion était équipé d'ordinateurs (en vue de notes) et d'enregistreurs audio. 
L'expérience montre que «... la quantité d'informations obtenues en quelques heures de réunion d'analyse collective du travail est impressionnante». De plus, «les informations apparaissent de l'intérieur d'un contexte, déjà polies par les expériences collectives et individuelles. En quelques heures, on extrait de la routine du travail ce qu'elles ont d'essentiel et de commun. » (Ferreira \& Iguti, 1996, p. 147).

Chaque réunion a été enregistrée et transcrite. Au bout du cycle de réunions, il y avait près de 26 heures d'enregistrement reportées qui ont servi de base pour construire et systématiser la description du travail des inspecteurs de la Receita Federal et des conditions dans lesquelles il se déroulait, selon la perception des participants. La transcription de ces entretiens a été analysée en utilisant la méthode d'analyse de contenu (Bardin, 1995). Dans les descriptions faites par les inspecteurs au sujet de leur travail, les chercheurs ont trouvé des thèmes, des idées ou des catégories, qui ont aidé à organiser et à analyser leurs affirmations. Les thèmes récurrents, ressortis des entretiens, ont ensuite été regroupés selon leur similarité et rassemblés dans un rapport préliminaire.

Il était méthodologiquement fondamental que le rapport produit soit critiqué, perfectionné et validé par les participants du processus. La validation a eu lieu en deux étapes. Lors de la première, des représentants du syndicat ont évalué les rapports préliminaires et suggéré des modifications qui visaient surtout à préserver l'anonymat des participants et les informations confidentielles. Des changements dans les textes préliminaires ont été effectués et les versions modifiées soumises aux participants des réunions, dans la seconde phase. C'est ici que les rapports ont été examinés par les participants de chaque réunion, individuellement et, ensuite, dans des réunions de validation. Ils ont subi les changements considérés comme nécessaires par les enquêteurs et les enquêtés présents, quelques suggestions par écrit étant, elles aussi, prises en compte. Les récits particuliers à chaque groupe étudié, après une révision de l'équipe des chercheurs, ont enfin été condensés dans un seul rapport.

\section{4.- Résultats}

Lorsqu'un collectif de travailleurs décrit et cherche à comprendre son activité avec la participation d'enquêteurs, la révélation spontanée de contenus subjectifs, reliés les uns aux autres, donne une signification et une logique à l'expérience vécue (Clot \& Faïta, 2000, p. 9). Cette révélation spontanée peut parler de souffrances, de défenses collectives et de plaisirs si le travail est ainsi vécu.

Les chercheurs se sont aperçus que les inspecteurs connaissaient des moments difficiles, caractérisés par des conflits, des incertitudes, des paradoxes et des malaises. Ces vécus sont des conséquences collectives de l'organisation du travail avec, à maintes reprises, un empiétement sur la vie personnelle. Comme l'a dit un inspecteur de la Receita Federal : "Ça fait vingt ans que je travaille à la Receita et j'ai vu tout son déclin. J'ai fini par quitter mon lieu de travail car je n'avais plus de santé. Si vous voyiez... dans la Receita, il y a un grand pourcentage de diabétiques, de gens souffrant d'hypertension... ».

« À l'aéroport, dix personnes sont mortes d'infarctus. Coïncidence ?! »

« Alors, en réalité, il faut voir comme ça. Elle a dit qu'elle est fière de la Receita Federal, mais on voit certains qui ont hâte d'y rentrer et nous qu'y sommes, nous avons hâte de partir. Il y a vingt ans, personne ne pensait à quitter la Receita Federal »

L'enquête a montré diverses facettes de la relation des inspecteurs avec leur travail, ne convergeant pas nécessairement : elles peuvent exprimer différentes manières de regarder et de laisser manifester les contradictions de l'organisation du travail. Nous présenterons des résultats et dans les considérations finales, nous les mettrons en relation avec les questions suivantes : le travail comme système d'organisation du pouvoir, l'obsession de la rentabilité financière, la dictature du temps réel, le risque, la peur, la souffrance et la solitude imposés par la logique du management (Dejours, 1980 ; Gaulejac, 2005b). 
La Receita Federal, de même que d'autres secteurs de l'administration publique et privée, a créé et implanté un système d'incitation à la performance par objectifs pour l'inspecteur. Ce système comprend une évaluation bimestrielle à l'occasion de laquelle les chefs notent leurs subordonnés. Le besoin d'atteindre les objectifs de recouvrement de l'impôt constitue non seulement un paradoxe en soi - puisqu'il n'est pas possible de "produire » le délit qu'on doit contrôler -, mais il a aussi crée une pression sur la profession en déplaçant le centre du travail vers des activités répondant au système d'évaluation, bien que distantes de la réalité. Un inspecteur a commenté à cet égard : "Seuls ceux qui ont à disposition quelque chose à transformer peuvent produire. Si je ne l'avais pas sous les mains, je ne pourrais pas produire. Je ne peux pas dire à quelqu'un de produire des illégalités pour avoir plus de contentieux à faire et maintenir une productivité élevée. C'est fou, ça !».

Le travail de quelques-uns peut ne pas s'appuyer sur le modèle imposé par le système d'évaluation, quoiqu'il repose sur un modèle de normalité cohérent avec la demande. En outre, la sollicitation d'informations par des services extérieurs à la Receita Federal (Ministère Public et Justice Fédérale) s'est accrue en augmentant la pression et le stress, car y répondre peut porter préjudice au respect des délais et des objectifs. « Eh bien, ma journée est une contrainte totale, parce qu'il y a beaucoup de choses à faire en peu de temps. Le pire c'est l'excès de rapports à faire et d'information à transmettre. En fait, c'est l'accumulation de beaucoup de données dans un court espace de temps de travail ».

Les inspecteurs ont manifesté des critiques à la mise en place d'un système informatisé de recouvrement. Bien que souhaitable, il aurait favorisé une bureaucratisation des procédures par une restriction de la créativité, de la liberté, de l'usage du bon sens et de l'expérience de la fonction. La rigidité des systèmes informatisés aurait en outre créé des limites dans le traitement de situations étrangères à des modèles préétablis. Par exemple, dans le domaine douanier, les horaires réduits d'accès au système poussent les inspecteurs vers un travail réalisé à la hâte, en les empêchant d'approfondir leurs analyses, même si la situation le requiert. Dans le domaine douanier, s'ajoutent aux difficultés du travail opérationnel les horaires imposés par le système informatique, dans la mesure où les auditeurs-inspecteurs doivent se presser pour tout accomplir dans les délais permis. Il s'agit d'un facteur de dépréciation de la fonction d'auditeur-inspecteur, car, «toujours en train de courir, on perd l'autorité de juger, par le système qui nous montre la vitesse à laquelle les choses doivent être traitées et c'est comme ça dans toutes les unités, la personne a vingt minutes ou une demie heure pour exécuter une opération ».

Le manque d'informations techniques et l'incommunicabilité entre programmes entraînent un risque d'erreur, engendrant chez eux une incertitude. La révision de certains dossiers n'est autorisée à personne d'autre que le responsable, de telle sorte que des décisions importantes sont prises en solitaire. Le sentiment que partagent les professionnels est qu'en adoptant le système informatisé, la haute administration a fait un choix politique et administratif en faveur de la centralisation et s'est servi de ce procédé pour restreindre le champ d'action des inspecteurs, et ce, pas seulement pour moderniser leurs conditions de travail.

Le risque de commettre une erreur face au souci de respecter les objectifs en temps réel, logique du système implanté, s'accompagne de la peur d'être puni par la Corregedoria (organisme responsable de la fiscalisation de l'exercice publique) qui, selon les inspecteurs des impôts, n'évalue pas toujours le dossier ayant mené à l'erreur du fonctionnaire. Ainsi, dans nombreuses occasions, l'erreur n'est pas le fruit de la mauvaise foi, mais le résultat d'une défaillance des conditions de travail. La Corregedoria est perçue comme étant plus encline à punir qu'à tirer les anomalies au clair. La peur des punitions, de la perte de l'emploi et des difficultés de défense menace leur activité. Ils craignent que, même en agissant correctement dans le cadre des règles, ils soient l'objet d'enquêtes, ce qui serait un facteur de gêne indépendamment du dénouement. 
Un autre aspect lié à la quantophrênie concerne la qualification et la formation professionnelle, ainsi que la diffusion des connaissances. D'importantes lacunes sont apparues dans la formation et dans la qualification à propos des normes, de l'informatique, des critères de prise de décisions, des paramètres techniques, etc. Les contrôleurs-inspecteurs soulignent qu'il existe un manque de formation, en particulier, dans l'utilisation des systèmes informatiques. Même s'ils se considèrent qualifiés, ils estiment qu'il serait nécessaire de suivre une formation et plus encore qu'on ne les sollicite pas pour des fonctions qu'ils ne sont pas toujours prêts à accomplir. Selon un contrôleur : «Je n'ai jamais fait aucune formation. Avec le système informatisé, on travaille comme autodidacte ». Les auditeurs-inspecteurs signalent un manque de manuels et modes d'emploi suffisants dans chaque domaine, ainsi qu'un manque de diffusion de leurs contenus. Il existe une procédure pour chaque type de service et, quand le contrôleur-inspecteur change de secteur, il emmène avec soi les connaissances, sans laisser de trace de la mémoire des connaissances de la procédure.

On a mentionné que la réalisation de formations dans ce service avait pour préalable que les participants agissent comme agents de la démultiplication des connaissances, ce qui ne fonctionnerait pas dans la pratique par inadéquation des profils requis. Les choix des candidats seraient également défectueux puisque les professionnels sélectionnés ne travaillent pas toujours sur le contenu présenté et qu'en fonction de ça, ils ne réussissent pas toujours à mettre en pratique le sujet appris. Parfois, cette désignation est basée sur la disponibilité ou encore sur le fait que le service en question ne reste pas dépourvu de représentant lors de la formation. Il peut arriver aussi que le secteur ne se prépare pas aux changements qui peuvent advenir de la formation et ainsi l'anéantir en conservant les habitudes antérieures ou en allouant le fonctionnaire récemment instruit à des activités qui se passent des connaissances acquises en cours.

On peut estimer que la formation qui précède la prise de fonction dans le service devrait mieux préparer les fonctionnaires à affronter à des situations de pression psychologique, telles que travailler sur des sommes importantes d'argent et être la cible de menaces en raison d'activités qui s'opposeraient aux intérêts économiques.

L'administration centrale s'est rendu compte qu'elle contribue souvent à transmettre une image négative de ce secteur ministériel et de ses agents auprès de la société. Le recul de l'autonomie et de l'autorité de ces derniers se traduit, au gré de la politique administrative, dans la façon dont ils sont vus et aussi dont ils se voient eux-mêmes, comme soumis à la dévalorisation personnelle et institutionnelle. Les témoignages de certains des contrôleurs montrent que le nouveau modèle de gestion, en plus de renforcer les relations asymétriques de pouvoir, provoque un conflit lié à une concurrence qui stimule des divisions internes :

\footnotetext{
«J'ai senti de la part de l'administration, qu'ils travaillaient pour que les contrôleurs et les techniciens ne s'entendent pas. Ils les montaient les uns contre les autres ».

«Dans certains cas, le patron vous ordonne de signer un document, vous n'êtes pas d'accord, mais il insiste. Personne ne vous apprend que vous n'êtes pas obligé de signer. J'ai vécu une situation dans laquelle je devais signer trois documents et ce fut une énorme pression. J'ai passé par quatre niveaux administratifs et je n'ai pas signé. On peut affronter ce type de situation, mais on n'est pas entrainé pour ça ».
}

Des récits de discrimination entre catégories professionnelles ont été entendus, tandis que les chefs étaient cités comme appartenant à un groupe tenu pour supérieur et qui se percevait ainsi. Par ailleurs, la catégorie professionnelle met l'accent sur la disproportion entre les gratifications destinées aux chefs de service et les grandes responsabilités liées aux attributions de l'inspecteur. Cela dénoterait de la part de l'administration supérieure, le peu de valeur accordée à celui qui assume effectivement des responsabilités.

Tous ces aspects réunis interviendraient directement dans l'appréciation portée par les chefs sur leurs subordonnés, tantôt bienveillants avec la situation et émettant un jugement 
favorable reconnaissant les mérites des agents, tantôt se servant d'un jugement négatif pour punir les employés. On a constaté que surviennent souvent, des situations dans lesquelles il existe un fossé entre ce qu'il était prévu de faire et ce qui est effectivement réalisé. «L'emploi de détours », représente, en vérité, l'initiative d'en corriger les défauts et peut refléter, selon Detiene et Vernant (1974), une source essentielle de l'intérêt porté et selon Dejours et ses collègues (1994), peut se situer au cœur du plaisir pour le travail. Autrement dit, la désobéissance apparente et le « détour » révèlent l'envie de satisfaire le besoin de faire quelque chose de non prévu dans les procédures formelles, mais peuvent engendrer des conséquences professionnelles négatives.

L'inspecteur ne connaît pas toujours le travail de son collègue relié au sien, ce qui proviendrait peut-être d'une absence de communication, où interviennent le manque de confiance dans le travail de l'autre ainsi que la production de tâches doublées ou divergentes entre elles, ce qui permet une contestation des contribuables. Le défaut d'interaction complique la quête de solutions à des problèmes qui peuvent être courants. L'inspecteur a l'impression de travailler isolé, dépourvu de temps et d'espace pour échanger des connaissances et rechercher des solutions à des questions techniques. Le besoin de générer des résultats immédiats au service de recouvrement dénaturerait sa fonction et contribuerait à des erreurs et anachronismes dans le service. Les inspecteurs ont fait remarquer la présence d'une série de facilités légales destinées au contribuable, des aspects de la modernisation pour le développement du pays, mais qui, en réalité, restreignent l'exercice de leur fonction et de leur autorité.

Dans cette optique, il est compréhensible que les inspecteurs n'assimilent pas la Receita Federal à une institution dotée d'une mission claire, telle qu'ils l'ont vécue naguère. En conséquence, beaucoup ne trouvent pas en eux-mêmes une identité qui leur donnerait de l'importance dans la société. Cela étant, le travail n'est pas vécu avec satisfaction puisqu'il n'est pas socialement reconnu. L'actuelle absence de fierté débouche donc sur la réflexion et l'abattement.

Le besoin pour la Receita Federal de diffuser les travaux qu'elle réalise en insistant sur leur importance pour la société a été clairement souligné. C'est ainsi qu'à leur avis, la vision positive du travail de la catégorie professionnelle serait renforcée si l'image nourrie par la société à leur égard était régénérée, avant qu'elle ne se perde dans les changements de la politique administrative.

Le travail est considéré comme risqué à cause des pressions diverses et des menaces de violence et même de mort. Il faut comprendre que la peur accompagne ce péril. Le risque relaté déborde le risque administratif et comprend les autres, physiques et psychologiques. Ils se sentent vulnérables et rejetés et en attribuent la responsabilité au service qui, éloigné de leur réalité, se désintéresse des problèmes quotidiens qu'ils affrontent. Un inspecteur a dit : «Si quelqu'un fait une erreur, l'administration punit cette personne. Il faut travailler et encore subir le risque d'être puni. L'employé irresponsable ne signe pas n'importe quoi et n'est pas puni, à l'exception d'une réduction de sa rémunération. Et le bon employé en plus d'effectuer toutes les tâches doit encore agir en tant que baby-sitter pour ces personnes. Il est très difficile de travailler dans les services internes. $S$ 'il s'agit d'un service externe, vous prenez soin de vous et vous accomplissez ce qui vous a été attribué ». Selon un autre inspecteur : "c'est un métier avec beaucoup de risques. Juste un chiffre : un inspecteur des impôts est tué tous les deux ans. Assassiné. C'est un risque que nous courons... un risque très élevé. (...) Il y a aussi les attaques et les menaces. Il existe des cas de collègues qui ont été déplacés en raison de menaces ».

La souffrance côtoie leur travail et s'exprime diversement par le découragement, le sentiment d'échec, le désenchantement, la ségrégation, la peur du danger, du contribuable armé, de l'insécurité, de la solitude et de l'isolement occasionnant une basse estime d'euxmêmes. Ce malaise se manifeste sous la forme d'insomnies, de plaintes et d'angoisses. 
Le déchirement d'avoir à contrôler le travail d'un collègue apparaît également comme une souffrance qui se répercute sur l'unité du travail sous forme de problèmes de gestion de l'organisation des tâches et de gestion des ressources humaines. En contrepartie, ils soulignent leur goût pour leur activité qui, malgré certaines situations délicates, peut contribuer à l'amélioration de la société.

Selon Dejours et ses collègues (1994), quand il y a un affrontement du danger entraînant l'expérience de la peur, les mécanismes de défense peuvent être déclenchés, ce qui fait que l'individu, au collectif, développe une négation du péril dans l'exercice de la fonction. Ces mécanismes suivent les individus et causent des effets réels aussi bien sur leur productivité que sur leur santé, au moins provisoirement. Ils cooptent ceux qui entrent, autrement dit les «nouveaux» qui doivent s'adapter à eux, de façon à ce que ce cycle ne se rompe pas. Le problème repose sur le caractère transitoire et éphémère de cet effet. Lorsqu'il cesse de fonctionner, il peut mener, dans ces lieux, à un appauvrissement émotionnel, un isolement et un affaiblissement des rapports sociaux, entraînant de la souffrance, des maladies psychosomatiques et menant à des congés maladie.

La demande de congé maladie est envisagée par quelques-uns comme un moyen d'évasion d'une occupation épuisante ou risquée. Quand des inspecteurs prennent des congés, le travail s'accumule pour ceux qui restent en activité, avec des impacts négatifs sur leur santé. Un cercle vicieux lié à la répartition des ressources humaines et qui ne peut trouver d'issue en lui-même, s'établit alors : une gestion est requise qui englobe le plan de carrière, la politique de salaires, la mutation, etc. Cette spirale est nettement défavorable au travail, à son résultat et à l'individu. Il faut comprendre que ces mécanismes sont fondés sur la vie et la culture de cette organisation particulière.

L'enquête a signalé que l'expérience de la peur fait naître des mécanismes de défense qui se développent et se mettent en place pour protéger les inspecteurs de la situation qu'ils vivent. Les menaces et la peur qui résultent décourage l'exécution du travail. Ceux-ci se plaignent d'être traités comme des suspects par la législation et par leurs chefs.

En dépit des problèmes et des frustrations, les inspecteurs se sentent concernés par leur travail, bien qu'ils remarquent que des collègues n'œuvrent pas comme ils le devraient. Beaucoup considèrent que les congés maladie sont parfois employés comme une tactique que le fonctionnaire adopte pour s'esquiver. Un cas est cité où le congé maladie, le congé d'ancienneté et le congé annuel sont apparus comme des stratégies du subordonné pour échapper à son déplacement vers un poste déterminé qu'il ne souhaitait pas occuper. En revanche, quand les problèmes de santé sont véritables, les chefs ne trouvent pas toujours d'appui dans l'administration supérieure dans la recherche d'une façon d'aider leurs subalternes.

On remarque que les inspecteurs, même si diffusément, relatent que l'institution ne devrait pas négliger la santé des employés, dès lors que l'apparition de la maladie peut être en rapport avec le travail.

\section{5.- Considérations finales}

L'enquête a révélé que des congés médicaux constituaient des stratégies individuelles de défense, destinées à préserver la santé physique et mentale face à l'impact de profonds changements survenus dans l'organisation du travail. Les résultats de l'étude sont devenus des outils au service d'une stratégie de défense collective de cette catégorie professionnelle, tracée par le syndicat UNAFISCO - Rio. Dans des occasions postérieures, la recherche a fourni des arguments pendant les conflits et les négociations entre le syndicat et la haute direction de la Receita Federal.

Le nouveau modèle de gestion du processus de travail des inspecteurs de la Receita Federal engendre un malaise dans la profession. Il est devenu évident que le service ne satisfait plus 
les attentes d'un grand nombre de fonctionnaires, malgré la stabilité de l'emploi et que la cooptation visée par le discours de modernisation et de qualité, n'opère plus. L'absence de satisfaction dans le travail est accompagnée de manque de motivation, de participation et d'engagement provoquant d'innombrables anachronismes, cédant leur place à de nombreuses interprétations isolées, faute d'explications rationnelles. La plainte sur le manque de notion d'ensemble, en particulier, révèle un morcellement du travail qui lui soustrait sa signification. Ceci peut être résumé dans les mots d'un inspecteur: «Tu découvres que cette montagne de travail, générant de la productivité, n'avait pas besoin d'être accomplie et que c'étaient des bêtises ».

Une importante implication pour les inspecteurs de la Receita Federal identifiée lors de la recherche est que l'obsession pour les résultats, c'est-à-dire le recouvrement, a transformé l'image de leur profession et leur propre identité professionnelle et sociale. Tenue par beaucoup d'entre eux comme dotée de la mission de faire respecter la justice fiscale, la Receita Federal n'est plus assimilée à une institution au service de la nation. Une part importante de l'insatisfaction ressentie semble liée à un sentiment de dévalorisation et à l'absence d'une politique de ressources humaines adéquates. Leur méfiance est liée en particulier à la mise en place de politiques de centralisation et de façon non participative, d'objectifs et de mesures de performances et d'évaluation qui ne correspondent pas à la réalité.

Il existe une forte convergence des témoignages dans la critique à la manière dont la quantification du travail se plie au temps réel dans ses tentatives d'atteindre les résultats prévus. Tous les processus nouveaux mis au service de la productivité sont envisagés avec défiance par la profession qui les perçoit comme des limites à ses capacités, ses connaissances et ses expériences. Il est également possible d'observer une ressemblance entre les résultats obtenus dans cette recherche et les résultats d'études réalisées avec d'autres catégories professionnelles, comme les pilotes d'avion (Ferreira et al., 1992), les professionnels d'un département de recherche industrielle (Molinier, 2012) ou les professeurs d'école (Maranda, Viviers, \& Deslauriers, 2013).

Gaulejac (2005b, p. 97) en désignant la quantophrênie comme une maladie de manager, avance que «les "calculocrates" préfèrent l'illusion de la garantie plutôt qu'une réalité remplie d'incertitudes, qui fait peur ». Dans cette optique, les aspects soulignés par Dejours quant à la recherche de résultats rapides au détriment de la qualité de vie et de la santé du travailleur affaiblissant mentalement son corps de métier, sont évidents.

Dans un travail plus récent publié au Brésil, Dejours et Ramos (2012) indiquent que la souffrance éthique peut ouvrir un nouveau chapitre dans la clinique du travail qui « rend plus compréhensible une deuxième tendance relative à la manière dont notre échelle sociale de valeurs est prise en compte, à savoir, le jugement que le sujet fait de lui-même, non seulement de la qualité de sa contribution par rapport à la production, mais de sa valeur éthique (...) parce que, par son activité productive, le travailleur compromet, en effet, le sort de l'autre, en particulier du client, dont il est recommandé de tromper, ou du subordonné, dont il est enjoint de maintenir sous pression ».

La recherche indique comment les conséquences perverses des actions promues par les «calculocrates» se radicalisent dans la souffrance éthique pouvant même aboutir en une maladie addictive, qui engendre des liens de dépendance dans le corps et l'âme. C'est ce dont nous a parlé un inspecteur dans son témoignage : «je pense que la fiscalisation est une drogue, comme la cocaïne. À présent, pour quitter la fiscalisation, je devrais fréquenter une clinique pour me désintoxiquer. Tout fait très mal. Je ne dors pas. Le juge peut décréter l'arrestation d'un inspecteur si celui-là n'agit pas selon l'attente du juge, mais malgré ça, on finit par rester, c'est le vice.» 


\section{Bibliographie}

Bardin, L. (1995). L'analyse de contenu. Paris: PUF.

Brun, J.P. (1992). Les hommes de lignes : analyse des phénomènes sociaux et subjectifs dans l'activité de travail des monteurs de lignes électriques. Thèse de doctorat en ergonomie, École Pratique des Hautes Études, Paris.

Clot, Y., \& Faïta, D. (2000). Genres et styles en analyse du travail : concepts et méthodes. Travailler, $4,7-42$.

Cru D., \& Dejours C. (1983), Savoir-faire de prudence dans les métiers du bâtiment. Cahiers médicaux-sociaux, 27, 239-247.

Dejours, C. (1980). Travail : usure mentale (essai de psychopathologie du travail). Paris: Éditions du Centurion.

Dejours, C. (1995). Comment formuler une problématique de la santé en ergonomie et en médecine du travail ? Le Travail Humain, 58(1), 1-16.

Dejours, C. (1998). Souffrance en France : La banalisation de l'injustice sociale. Paris: Seuil.

Dejours, C. (2011). La psychodynamique du travail face à l'évaluation : de la critique à la proposition. Travailler, 25, 15-27.

Dejours, C., Abdoucheli, E., \& Jayet, C. (1994). Psicodinâmica do trabalho: contribuições da escola dejouriana à análise da relação prazer, sofrimento e trabalho. São Paulo: Atlas.

Dejours, C., \& Begue, F. (2009). Suicide et travail : que faire? Paris: PUF.

Dejours, C., \& Ramos, G.A. (2012). Psicodinâmica do trabalho e teoria da sedução. Psicologia em Estudo. 17(3), 363-371.

Detiene, M., \& Vernant, J.P. (1974). Les ruses de l'intelligence : la mètis chez les Grecs. Paris: Flammarion.

Ferreira, L.L. (1998). Análise coletiva do trabalho dos cortadores de cana da região de Araraquara (SP). Fundacentro, São Paulo.

Ferreira, L.L., Bussacos, M.A., Schlithler, C.R.B., Maciel, R.H., \& Miyashita, R. (1992). Voando com os pilotos: condições de trabalho dos pilotos de uma empresa de aviação comercial. Fundacentro, São Paulo.

Ferreira, L.L., \& Donatelli, S. (2002). Análise coletiva do trabalho de pescadores-mergulhadores de lagosta brasileiros. Fundacentro, São Paulo.

Ferreira, L.L., \& Iguti, A.M. (1996). O trabalho dos petroleiros: perigoso, complexo, contínuo e coletivo. São Paulo: Editora Scritta.

Gaulejac, V. (2005a). L'abstraction et la psychologisation du pouvoir dans l'entreprise hypermoderne, in J-C. De Crescenzo (Ed.), Changement dans les organisations. Tome 2 - Communication, négociation et interventions (pp. 11-24). Paris: L'Harmattan.

Gaulejac, V. (2005b). La société malade de la gestion : idéologie gestionnaire, pouvoir managérial et harcèlement social. Paris: Seuil.

Gaulejac, V. (2005c). Ne pas psychologiser les problèmes sociaux, échange avec Vincent de Gaulejac. Gestalt, 29(1), 188-192.

Gaulejac, V. (2008). Approche socioclinique de la souffrance au travail. Revue Internationale de Sociologie, 18(3), 433-441.

Gaulejac, V. (2009). Qui est «je»? Paris: Seuil.

Guyon, M. (2010). V. de Gaulejac. Qui est «je» ?, L'orientation scolaire et professionnelle[En ligne], 39/3, mis en ligne le 16 décembre 2010. URL : http://osp.revues.org/index2877.html, Éditeur: Institut national d'étude du travail et d'orientation professionnelle.

Hallack, F. S., \& Silva, C. O. (2005). A reclamação nas organizações do trabalho : estratégia defensiva e evocação do sofrimento. Revista Psicologia \& Sociedade, 17(3), 67-72.

Lacroix, M. (2004). Le développement personnel : du potentiel humain à la pensée positive. Paris: Flammarion.

Maranda M-F. (1995). La psychodynamique du travail, une alternative à l'individualisation de la santé mentale au travail. Revue Santé Mentale au Québec, 20(2), 219-242. 
Maranda M-F., Viviers, S., \& Deslauriers, J-S. (2013). L'école en souffrance : recherche-action sur les situations de travail à risque pour la santé mentale en milieu scolaire. Nouvelle Revue de Psychologie, 15, 225-240.

Marcuse, H. (1964). One-dimensional man: studies in the ideology of advanced industrial society. Boston: Beacon Press.

Molinier, P. (2012). Une enquête de psychodynamique du travail dans un département de recherche industrielle. Méthodologie, élaboration, résultats. Bulletin de Psychologie, 65(519), 217-227.

Perret, V. (2009). Quand le changement devient soluble ou l'idéologie managériale du changement organisationnel. In D. Golsorkhi, I. Huault, \& B. Leca, (Eds.), Critique du management : une perspective française (pp. 209-231). Sainte-Foy: Presses de l'Université de Laval.

Renault, E. (2008). Souffrances sociales : Philosophie, psychologie et politique. Paris: La Découverte.

Ribeiro, R.M.S. (1992). O trabalhador em ambiente de alta tecnologia. Thèse de doctorat. Instituto de Psicologia da Universidade de São Paulo - USP, São Paulo.

\section{RÉSUMÉ}

L'article présente les résultats d'une enquête menée auprès d'inspecteurs des impôts de la Receita Federal (l'administration fiscale) du Brésil dans l'État de Rio de Janeiro. Il s'appuie sur les contributions de Dejours (1999) et de Gaulejac (2005b) sur l'alignement de la gestion publique sur le management privé en soulignant l'insatisfaction et la souffrance de ces inspecteurs liées à des changements mis en place par la haute administration du service dans son projet « Moderniser le Brésil ».

\section{MOTS-CLES}

management et gestion publique au Brésil, analyse collective du travail, subjectivité dans le travail, travail des inspecteurs des impôts, insatisfaction des fonctionnaires

\section{REFERENCEMENT}

Bartholo, R., Monteiro Afonso, R. de C., Marques Campos, A.E., Bursztyn, G., \& Delamaro, M.C. (2015). Analyse collective du travail des inspecteurs des impôts au Brésil dans 1' État de Rio de Janeiro. Activités, 12(1), 104-117. http://www .activites.org/v12n1/v12n1.pdf

Article soumis le 01/10/2013, accepté le 14/02/2015 